\title{
Fate of Extruded Sealer: A Matter of Concern
}

\author{
Ajay Chhabra ${ }^{1}$, Tanvir S. Teja ${ }^{2}$, Varun Jindal ${ }^{3}$, Meenu G. Singla ${ }^{4}$, Karan Warring ${ }^{5}$
}

\begin{abstract}
The most important objective of successful root canal treatment is thorough biomechanical preparation of root canal. Elimination of infected pulp and dentine, adequate root canal preparation and three dimensional obturation constitute the basic principle of root canal treatment. Ideally, the filling material along with sealer should be confined to the root canal without extending to periapical tissue or other neighboring structures. Endodontic filling material and sealer, beyond the apical foramen may give rise to clinical manifestations as a result of the toxicity of the product. When the extruded material is either close to or in intimate contact with nerve structures, anesthesia, hypoaesthia, paraesthasia, or dysaesthesia may occur. The purpose of this paper is to discuss few cases of apical extrusion of sealer during obturation and its effects on periapical tissue and the success of treatment.
\end{abstract}

Keywords: Extruded sealers, Zinc oxide Eugenol Sealer, Obturation, Lateral condensation, Resorption

${ }^{1}$ HOD \& Professor

Department of Operative \& Endodontics

Bhojia Dental College, Baddi,

Himachal Pradesh,

INDIA

${ }^{2}$ Reader

Department of Operative \& Endodontics

Bhojia Dental College, Baddi,

Himachal Pradesh,

INDIA

${ }^{3}$ Senior Lecturer

Department of Operative \& Endodontics

Bhojia Dental College, Baddi,

Himachal Pradesh,

INDIA

${ }^{4}$ Professor

Dept. of Conservative Dentistry \& Endodontics

Sudha Rustagi College of Dental Sciences \&

Research, Faridabad, Haryana,

INDIA

${ }^{5}$ Post Graduate Student

Department of Operative \& Endodontics

Bhojia Dental College, Baddi,

Himachal Pradesh,

INDIA

\section{INTRODUCTION}

$\mathrm{R}$ oot canal sealer plays an important role in the obturation of the root canal. The sealer fills all the spaces the gutta percha is unable to fill because of gutta-percha's physical limitations.

Sealer helps to fill in irregularities and minor discrepancies between the filling and canal walls, accessory canals and multiple foramina. Sealer discloses the presence of resorptive areas, root fractures, shape of the apical foramen and other structures due to its radio-opacity. The sealer acts as a binding agent to the dentin and to the core material, which usually is gutta percha.

A sealer is a good lubricant. Thus, helps in the seating of primary cone into the canal. It is a good germicidal or antibacterial. The sealer is usually a mixture that hardens by chemical reaction, such reaction normally includes the release of toxic material, making the sealer less biocompatible (1, 2).

Thus, it is important that sealer and obturating material should be confined in the root canal system.

According to Grossman, ideal sealer should be tacky when mixed, to provide good adhesion between it and the canal wall when set, make a three dimensional fluid tight seal, have ample setting time, should not shrink upon setting and not stain tooth structure. It should be bacteriostatic, be insoluble in tissue fluids and be tissue tolerant $(1,3,4)$.

The periradicular tissue reaction after root canal treatment depends on the physical adequacy and biocompatibility of the obturation material along with other factors such as the preexisting disease, the elimination of disintegrating pulp tissue by meticulous access preparation, debridement, and shaping of the root canal system; the distance from the apical foramen(or foramina) $(3,5,6)$.

\section{EUGENOL BASED SEALER CEMENT}

Many root canal cements are based on zinc oxide eugenol, which is known to provide a good seal. Many of these sealers are simply zinc oxide eugenol cements that have been modified for endodontic use. The mixing vehicle for these materials is mostly eugenol. The powder contains zinc oxide that is finely sifted to enhance the flow of the cement. Setting time is adjusted to allow for adequate working time.

One millimeter of zinc-oxide eugenol cement has a radio-opacity corresponding to 4-5 mm of aluminum, which is slightly 
lower than gutta-percha. These cements easily lend themselves to the addition of chemicals and para-formaldehyde is often added for antimicrobial and mummifying effects, germicides for antiseptic action, rosin or Canada balsam for greater dentin adhesion, and corticosteroids for suppression of inflammatory reaction $(3,7)$.

Zinc oxide eugenol sets because of the combination of chemical and physical reaction, yielding a hardened mass of zinc oxide embedded in a matrix of a long sheath like crystals of zinc eugenolate $\left[\mathrm{C}_{10} \mathrm{H}_{11} \mathrm{O}_{2}\right]^{2} \mathrm{Zn}$. Excess eugenol is invariably present and is absorbed by both zinc oxide and eugenolate. The presence of water, particle size of the zinc oxide, the $\mathrm{pH}$ and the additives are all important factors in the setting reaction (4). Hardening of the mixture is due to zinc eugenolate formation; unreacted eugenol remains trapped and tends to weaken the mass.

All zinc oxide eugenol cements have an extended setting time but set faster in the tooth than the glass slab, due to increased body temperature and humidity. If the eugenol used in Grossman's cement becomes oxidized and brown, the cement sets too rapidly for ease of handling. If too much sodium borate has been added, the setting time is overextended (7).

The original zinc oxide-eugenol cement, developed by Rickert's was the standard for the profession for years. It admirably met the requirement set by Grossman for
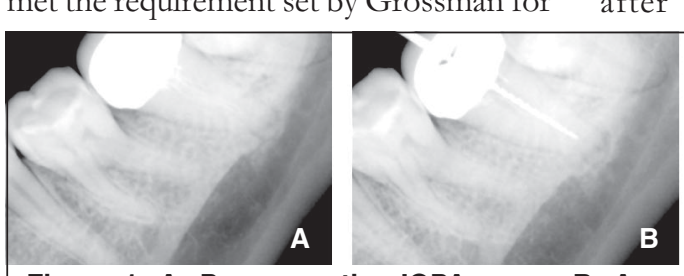

severe staining. The silver, added for radiopacity, causes discoloration of the teeth, thus creating an undesirable public image for endodontics. Removing all cement from the crowns of teeth would prevent these unfortunate incidents (8).

In 1958, Grossman recommended nonstaining zinc oxide eugenol cement as a substitute for Rickert's formula. It has become the standard by which other cements are measured because it reasonably meets most of Grossman's requirements for root canal sealer (9).

The most common zinc oxide eugenol cements are Rickert's sealer, Grossman's sealer, Wach's paste, Tubliseal. The purpose of this article is to present a series of case reports in which the sealer had extruded beyond the apical foramen causing discomfort to some patients and to discuss the fate of the sealer.

\section{CASE 1}

A 28 year female patient reported with pain in left mandibular back region to the Department of Conservative Dentistry and Endodontics, Bhojia Dental College and Hospital, Budh, Baddi. Post operative XRay showed mesio angular impaction along with RCT done on mandibular left 2nd molar with porcelain crown fixed on it. On clinical examination mandibular 2nd molar was tender on percussion. Re- treatment was then planned for the lower 2nd molar. Disinfection and biomechanical preparation was done. Patient was recalled after 5 days and was asymptomatic.
Obturation was done with lateral condensation method and Grossman sealer was accidentally pushed into the canal. Post operative radiograph showed sealer in the periapical area. Patient was advised anti inflammatory drugs only if required. Post operative pain was observed, which subsided in 3 days. The patient reported back after 2 months. Intra oral periapical radiograph showed complete resorption of the extruded sealer.

\section{CASE 2}

A 33 year old male patient reported to the Department of Conservative Dentistry and Endodontics, Bhojia Dental College and Hospital, Budh, Baddi, with pain in his lower left mandibular region. Root canal treatment was performed for his mandibular second premolar and first molar utilizing lateral compaction technique with gutta percha and Tubliseal sealer. Peri- apical radiograph showed lateral extrusion of the sealer in premolar region and extrusion of the sealer in the periapical region in the molar. After three weeks, resorption of sealer was seen in the premolar periapical region which was then restored with post and core. Similarly, molar region showed extruded sealer which resorbed subsequently and no pain was observed in examination.

\section{CASE 3}

A 52 year old female patient was referred to the Department of Conservative Dentistry and Endodontics, Bhojia Dental College and Hospital, Budh, Baddi from the

Figure 1: A: Pre- operative IOPA X- ray. B: Access opening made in the crown. C: obturation done. D,E: Peri - apical Xray shows extruded sealer which later resorbed

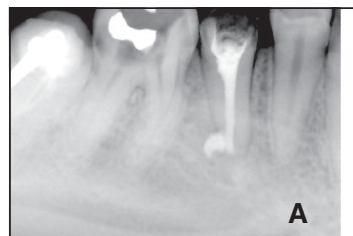

A

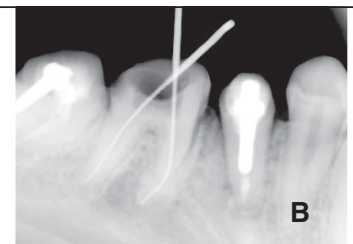

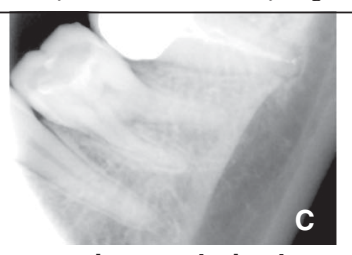
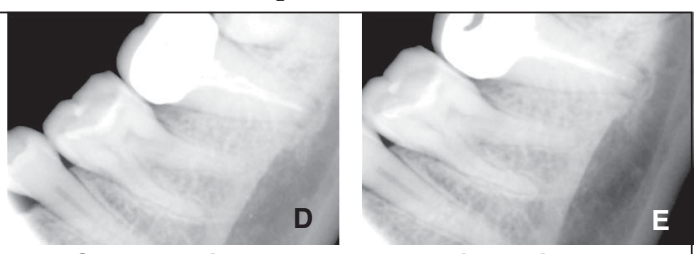

E

Figure 2: A: Pre- operative IOPA showing lateral extrusion of sealer. B,C: IOPA showing resorption after week with post and core. D,E: Peri - apical $x$ - ray showing extruded sealer in the molar, which later resorbed. 


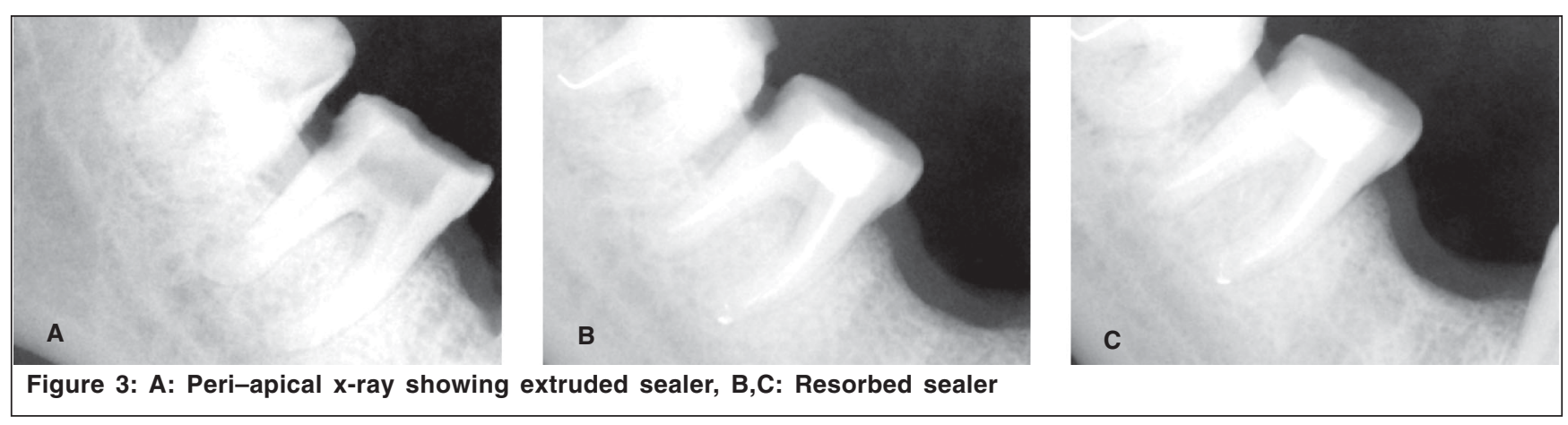

department of prosthodontics. The patient had a missing mandibular first molar. She was advised for a 3 unit fixed partial denture for which root canal treatment had to be performed on the second molar. Root canal treatment was done for the second molar using lateral compaction technique with gutta percha and Wach's sealer. Some of the sealer extruded beyond the apical foramen with some discomfort to the patient. Analgesics were prescribed to the patient. Post operative intra oral peri apical radiograph was taken after 2 weeks revealing complete resorption of the sealer.

\section{CASE 4}

Root canal treatment was performed for a 44 year old male patient who reported to our department with severe pain in right lower mandibular region. During obturation of mandibular second premolar and first molar with gutta perch and Tubliseal sealer, slight extrusion of the sealer was seen on the radiograph. Patient did not report of any post operative pain. After 3 weeks, follow up radiograph showed complete resorption of the sealer.

\section{CASE 5}

Root canal treatment was performed for a 32 year old female patient who reported to our department with severe pain in the

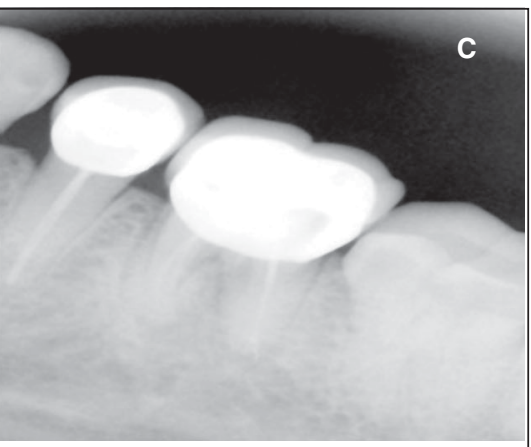

Figure 4 : A: Peri - apical x- ray showing Extruded sealer,B,C: Resorbed sealer
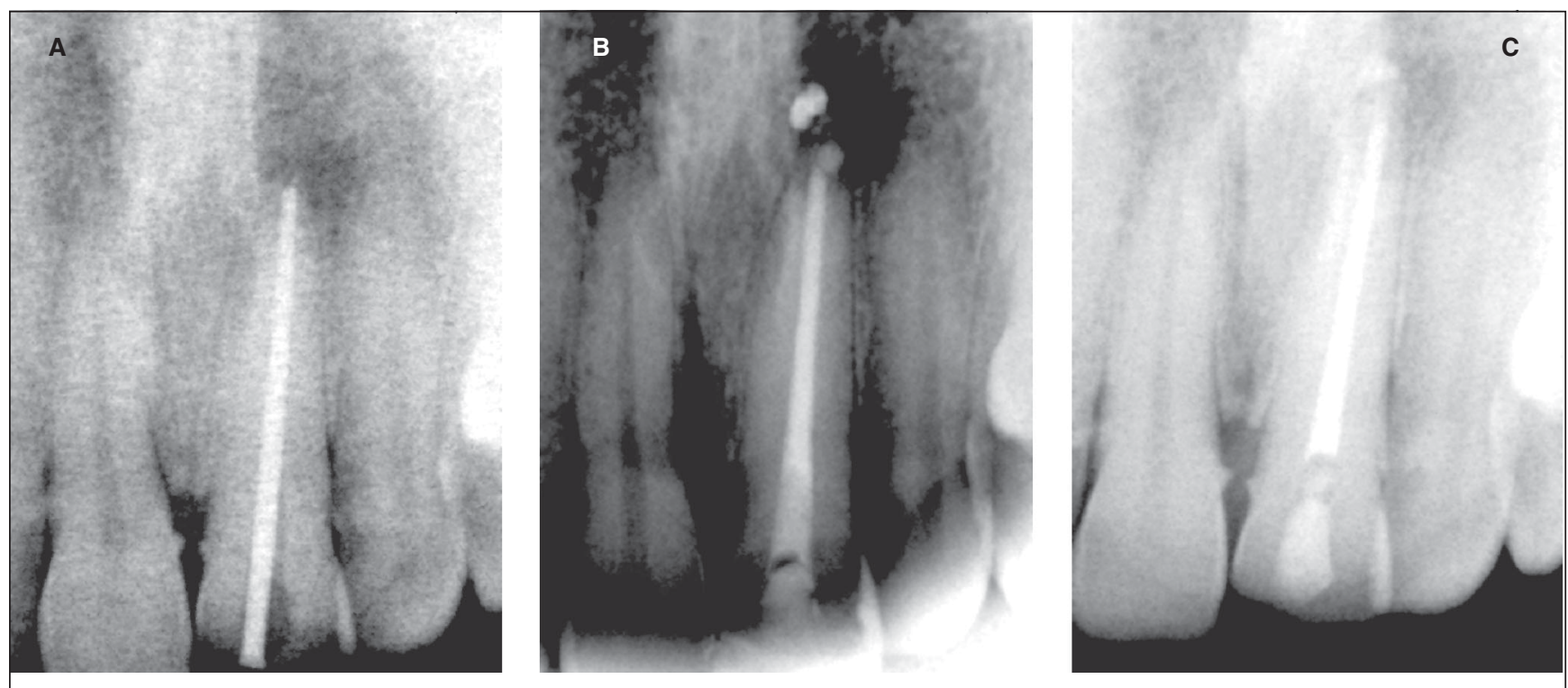

Figure 5: A,B : Peri - apical x- ray showing Extruded sealer,C: Resorbed sealer 


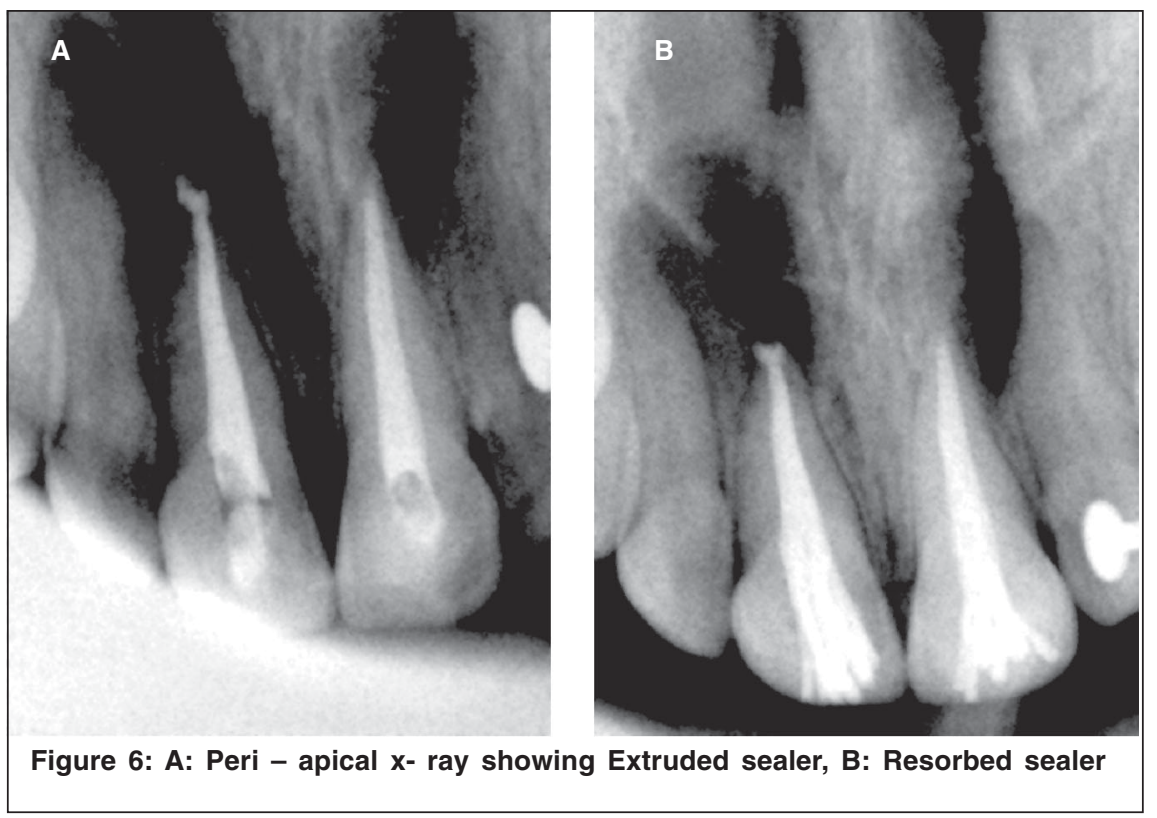

anterior maxillary region. During obturation of maxillary central incisior with gutta perch and Rickert's sealer, peri apical extrusion of the sealer was seen on the radiograph. Patient did not report of any post operative discomfort. After 3 months, follow up radiograph showed resorption of the sealer.

\section{CASE 6}

Root canal treatment was performed for a 33 year old female patient who reported to our department with pain in the anterior maxillary region. During obturation of maxillary central incisior with gutta perch and Wach's paste, peri apical extrusion of the sealer was seen on the radiograph. Patient did report of mild pain after the treatment. Analgesics were prescribed to the patient. After 3 months, follow up radiograph showed resorption of the sealer.

\section{DISCUSSION}

Cements, pastes, and solid core material have ideally been recommended for root canal filling or obturation. Usually a solid core material such as gutta percha cone is inserted into the root canal together with cement, a paste, or a solvent. However there is notable controversy in the literature, regarding the presence of cement beyond the apex.

From a medico-legal point of view, there are basically two questions to ask: What is the quantity of extrusion material which may be considered acceptable? According to the American Dental Association, overfilling by more than $2 \mathrm{~mm}$ past the radiological apex represents a technical error ascribable to over-instrumentation, inadequate measuring, or a lack of an apical stop. However the latter was difficult to obtain, as in the presence of resorbed roots caused by inflammatory processes or by particularly wide apices. Overinstrumentation, in particular, may extrude infected material contained in the canals beyond the apex, interfering, or impeding the healing process of the periapical tissue(8).

Various approaches are being used to evaluate scientifically the toxic effects of endodontic materials.

Cytotoxic studies have shown that almost all of today's sealers are toxic when first mixed, while they are setting over hours, days or weeks and some continue to age noxious elements for years. This is of course caused by dissolution of the cement thus releasing the irritants. For example, eugenol is not only cytotoxic but also neurotoxic $(10,11)$. As far as cytotoxicity studies are concerned one would have to rank the pure zinc oxide eugenol sealer as the worst followed Grossman's and
Rickert's sealer followed by Wach's, Tubuliseal, Sealapex CRS and finally Nogenol $(12,13,14)$.

Subcutaneous implants of root canal sealers, to test their toxic effects are done either by needle injection under the skin of animals or by incision and actual insertion of the product, either alone or in Teflon tubes or cups. Freshly mixed material may be implanted allowing it to set in situ or completely set material may be inserted to judge long term effects.

The sealers implanted directly into bone evoke less inflammatory response than the same cements evoke in soft tissue. From Marseille comes a report of two zinc oxide eugenol sealers implanted into rabbit's mandible. At four weeks both sealer implants showed "slight to moderate reactions -no bone formation, or bone resorption. At 12 weeks there was slight to very slight reactions - bone formation in direct contact with sealers and bone ingrowth into the implant tubes $(15,16)$. There is not enough evidence to rank cements implanted into bone.

Most ideal method of testing drug, a substance or a technique is in vivo in a human subject. But this is often dangerous, costly, unethical, and so animals are substituted. The closer ones to Humans are the monkeys.

Most root canal filling sealers produce an initial acute inflammatory reaction in the connective tissues. This is followed by production of chronic foreign body reaction in which mononuclear phagocytes and lymphocytes are prominent. As the material disintegrates into tissue fluid, particles are phagocytosed by macrophages. The activated macrophages elaborate enzyme such as collagenase which cause tissue destruction (17).

On comparing the various sealers, Erausquin and Muruzabal found that root canal overfilling depends to a certain degree, on the pressure applied to the material in the canal, the size of the foramen and the fluidity of the material. Disregarding the 
first two factors the fluidity of the cements depends both on the proportion of powder to liquid in the mixture and on the degree of spatulation. Periapical inflammatory reactions provoked by the root canal cements varied considerabely $(18,19)$.

These were the following observations drawn based on the various histological studies:

- Tested root canal cements showed good plasticity; no dimensional changes could be detected after filling was placed.

- Grossman, Kerr \& N2 permanent cements did not adhere to the canal walls; zinc-oxide eugenol was only slightly adherent; N2 temporary showed greatest adherence.

- Grossman, Kerr \& N2 permanent cements when mixed with debris, mostly provoked severe inflammatory infiltration, N2 temporary caused a mild reaction, Rickert sealer frequently induced moderate infiltration ingrowth of the periapical tissue, and resorption of the canal wall.

- Most favourable tissue reaction was found in specimen with fillings short of the apex and with minimal injury of the remaining pulp stump.

- All root canal cements tested, in case of overfillings showed a tendency to be resorbed. Resorption of compact overfilled masses, without debris proceeded slowly. No polymorphonuclear leucocytes were observed, although giant cells were nearly always found. When the extruded root canal sealers became mixed with tissue remnants, a severe inflammatory reaction was frequently seen.

- When the cements directly contacted the alveolar surface, necrosis and resorption of the superficial bone lamelae frequently occurred. However most severe resorptions were constantly associated with osteosclerosis of underlying bone marrow. This response was indirectly induced by the inflammation of the apical periodontal ligament, caused by poor debridement and filling of the canal

The extrusion of sealer through the apical foramen is an issue of concern. Some authors have stated that this may interfere with the repair process. The short-term clinical and radiographical follow-up revealed that only few of the cases were interpreted as endodontic failures. The remaining cases did not show postoperative complications, and no radiographic evidence of sealer was observed in the periapical tissues resulting in a return to a normal radiographic appearance. After regular follow up these cases appeared radiographically normal, indicating that the sealer was well tolerated by the periapical tissues. The few cases that showed a slight resorption of filling material within the lumen of the root canal had a root fill that was located approximately $2 \mathrm{~mm}$ from the radiographic apex. It was therefore believed that the sealer had disappeared, not the fill $(10,11,12)$.

There is no agreement, in fact, regarding the radicular level at which the treatment should reach, even though some metaanalyses have recognised that, over time, the best results for canal obturations occur when the gutta-percha arrives at 0-1 mm from the apex and, on the contrary, when considering measurements of greater than $1 \mathrm{~mm}$ (above or below the apex), the results are less favourable (20).

\section{CONCLUSION}

One must conclude that periradicular tissue reaction to all the cements will first be inflammatory, but as the cements reach their final set, cellular repair takes place unless the cement continues to break down, releasing one or more of its toxic components.

\section{REFERENCES}

1. Cohen S, Burns RC. Pathways of the pulp. 7th ed. St. Louis: Mosby Inc; 1998.

2. Grossman LV. An improved root canal cement. J Am Dent Assoc 1958;56:381.
3. Langeland K. Root Canal sealents and Pastes. Dent Clin North Am 1974;18:309.

4. Schilder H. Filling root canals in three dimensions. Dent Clin North Am 1967;723-44.

5. Lin LM, Langeland K. Light and electron microscopic study of teeth with carious pulp exposures. Oral Surg Oral Med Oral Pathol 1981;51:292-316.

6. Schilder $\mathrm{H}$. Cleaning and shaping the root canal. Dent Clin North Am 1974;18:26996.

7. Al Khatib ZZ, Baum RH, Morse DR, et al. The antimicrobial effect of various endodontic sealers. Oral Surg Oral Med Oral Pathol 1990;70:784.

8. Olsson B, Wennberg A. Early tissue reaction to endodontic filling materials. Endod Dent Traumatol 1985;1(4):138-41.

9. Heling I. The anti- microbial effect within dentinal tubules of four root canal sealers. J Endodon 1997;22:257.

10. Doblecki W, Turner DW. Leucocyte migration response to dental materials using Boyden chambers. J Endodont 1980;6:636.

11. Rodriguez H, Spangberg L, Langeland K. Biological effect of dental materials. Effect of zoe cements on Hela cells IN vitro. Estomatol Cult 1971;9:191.

12. Hanks CT, Anderson M, Craig RG. Cytotoxic effects of dental cements on two cell culture system. J Oral Path 1981;10:101.

13. Imai $Y$, Watanabi A. Evaluation of biological effects of dental materials using a new cell culture technique. J Dent Res 1982;61:1024

14. Lindquist L, Otteskog P. Eugenol liberation from dental materials and effect on human diploid fibroblast cells. Scand J Dent Rest 1982;8:120.

15. Becker RM, Hume WR. Release of eugenol from mixtures of zinc oxide eugenol in vitro. J Periodontol 1983;8:71.

16. Hume WR. An analysis of the release and diffusion through dentin of eugenol from zinc-oxide eugenol mixtures. J Dent Res 1981;63:881.

17. Salthouse TN, Matlaga BF. Microspectophotometery of macrophage lysosomal enzyme activity: A measure of polymer implant tissue toxicity. Toxicol Appl Pharmacol 1973;25:201.

18. Seltzer S, Turkenkopf S. Histological evaluation of periapical repair following positive and negative root canal cultures.Oral Surg 1964;17:507.

19. Erausquin J, Muruzabal M. Root canal filling with zinc oxide eugenol cements in rat molars. Oral Surg 1967;24:547.

20. Hensten-Peterson A, Helgeland K. Evaluation of biological effects of dentinal materials using different cell culture techniques. Scand $J$ Dent Res 1977;85:291. 